\title{
Die Lösse im Becken von Tafí (Nordwestargentinien) - ein Langzeitklima- archiv für das südamerikanische Monsunsystem?
}

\section{Andreas Schellenberger, Bern}

\section{Einleitung}

In der Quartärforschung sind nach wie vor grosse Wissensdefizite in der Südhemisphäre zu verzeichnen, wo grundlegende Fragen der Klärung harren. Auf dem südamerikanischen Kontinent ist dies vor allem einem Mangel an hochauflösenden und kontinuierlichen Langzeitklimaarchiven zuzuschreiben. Eine prominente Ausnahme stellen die Pollenprofile Funza I/II in der Hochebene von Bogotá (Kolumbien; ca. $5^{\circ} \mathrm{N}$ und $74^{\circ} \mathrm{W}$ ) dar, die zusammen das gesamte Quartär abdecken (HooghiEmSTRA 1984 und Folgearbeiten wie Hooghiemstra \& Ran 1994; Torres et al. 2005). Zwei Bohrkampagnen im Salar de Uyuni ermöglichten die Rekonstruktion der hygrischen Schwankungen auf dem bolivianischen Altiplano bis ca. 170 ka zurück (Fornari et al. 2001; FrITZ et al. 2004). Die meisten anderen in Südamerika durchgeführten Quartärstudien kommen jedoch nicht über den letzten Glazialzyklus hinaus, oder die zeitliche Auflösung der Sedimente ist zu grob, um detaillierte paläok limatische Aussagen treffen zu können (z.B. in den Pampa-Lössen). Infolgedessen sind zum gegenwärtigen Zeitpunkt die Entwicklung des Klimasystems und damit einhergehende Umweltveränderungen während des Früh- und Mittelpleistozäns weitgehend unbekannt. Zu klären bleibt insbesondere, ob der südhemisphärische Teil des Subkontinents den Klimasystemwandel der mid-Pleistocene transition (MPT; ca. 920 bis $640 \mathrm{ka}$ ) durchlaufen hat und ob er vor der letzten Warmzeit den gleichen hochfrequenten Klimaschwankungen unterworfen war, die in den Archiven der hohen Breiten dokumentiert sind.

Aus den südlichen Zentralen Anden ist jüngst eine $50 \mathrm{~m}$ mächtige Löss-Paläoboden-Sequenz beschrieben worden, die entscheidend zur Klärung dieser Fragen beitragen könnte (Abb. 1). In diesem, Las Carreras benannten Profil im Becken von Tafí in der nordwestargentinischen Provinz Tucumán wurde ein Basisalter der äolischen Sedimente von mindestens 1.2 Ma ermittelt (SCHELlenderger et al. 2003). Insgesamt 32 eingeschaltete Paläoböden spiegeln alternierende Trockenphasen (Löss-Ablagerung) und Feuchtphasen (Bodenbildung) wider. Auf der Grundlage der (pedo)faziellen Eigenschaften der Paläoböden und Lösse konnte die Sequenz in drei Bereiche (Units) unterteilt werden. Vergleichbar mit den chinesischen
Lössen, deren Potential zur Rekonstruktion der Klimaund Landschaftsgeschichte ausführlich beschrieben ist (Liu \& Ding 1998), belegt das Las Carreras-Profil bis in das Frühpleistozän zurückreichende Schwankungen des Paläoklimas (Schellenberger \& Veit 2006).

Mehrere Autoren haben die pleistozänen Feuchtphasen in den Zentralen Anden mit einer Nordverlagerung der Polarfront in Zusammenhang gebracht (z.B. Hastenrath 1971; Zinck \& Sayago 1999). Als Folge der damit einhergehenden Abschwächung der südostpazifischen Antizyklone sollen feuchte Westwinde aus den mittleren Breiten die relativ niedrigen patagonischen Anden überwunden und an der Ostabdachung der Anden für erhöhte Niederschläge gesorgt haben. Gegenwärtig liegt das Becken von Tafí im Einflussbereich des südamerikanischen Monsunsystems (ZHOU \& LAU 1998). Es stellt sich folglich die Frage, welches paläoklimatische Signal in den Tafí-Lössen abgebildet ist: ein tropisch-monsunales Sommerregenregime oder das Winterregenregime der mittleren Breiten (Westwindzone)?

Um diese grundsätzliche Frage zu klären, werden im folgenden zunächst die naturräumlichen und insbesondere die klimatischen Verhältnisse am Ostrand der Tucumaner Anden dargelegt. Auf dieser Grundlage werden anschliessend Argumente zusammengetragen, die eine Interpretation der Las Carreras Löss-Paläoboden-Sequenz als Archiv für den Paläomonsun stützen.

\section{Methoden}

Bei den in diesem Artikel angeführten Löss-Profilen handelt es sich um natürliche und künstlich geschaffene Aufschlüsse (Tab. 1). Sie wurden im Gelände eingehend beschrieben und anhand des pedostratigraphischen Aufbaus beprobt. Die Ansprache der Böden folgt der Food and Agriculture Organization of the United Nations (FAO), dem International Soll REFERENCE AND INFORMATION CENTRE (ISRIC) sowie der InTernational Society of Soll Science (ISSS) (1998). Zur Charakterisierung der Paläoböden und Lösse wurden im Labor bodenkundliche und sedimentologische Standardanalysen durchgeführt. Weitergehenden Fragestellungen, z.B. nach der Herkunft der Sedimente, wurde mit (isotopen)geochemischen, tonmineralogischen und mikromorphologischen Untersuchungen begegnet. Alle angewandten Methoden 


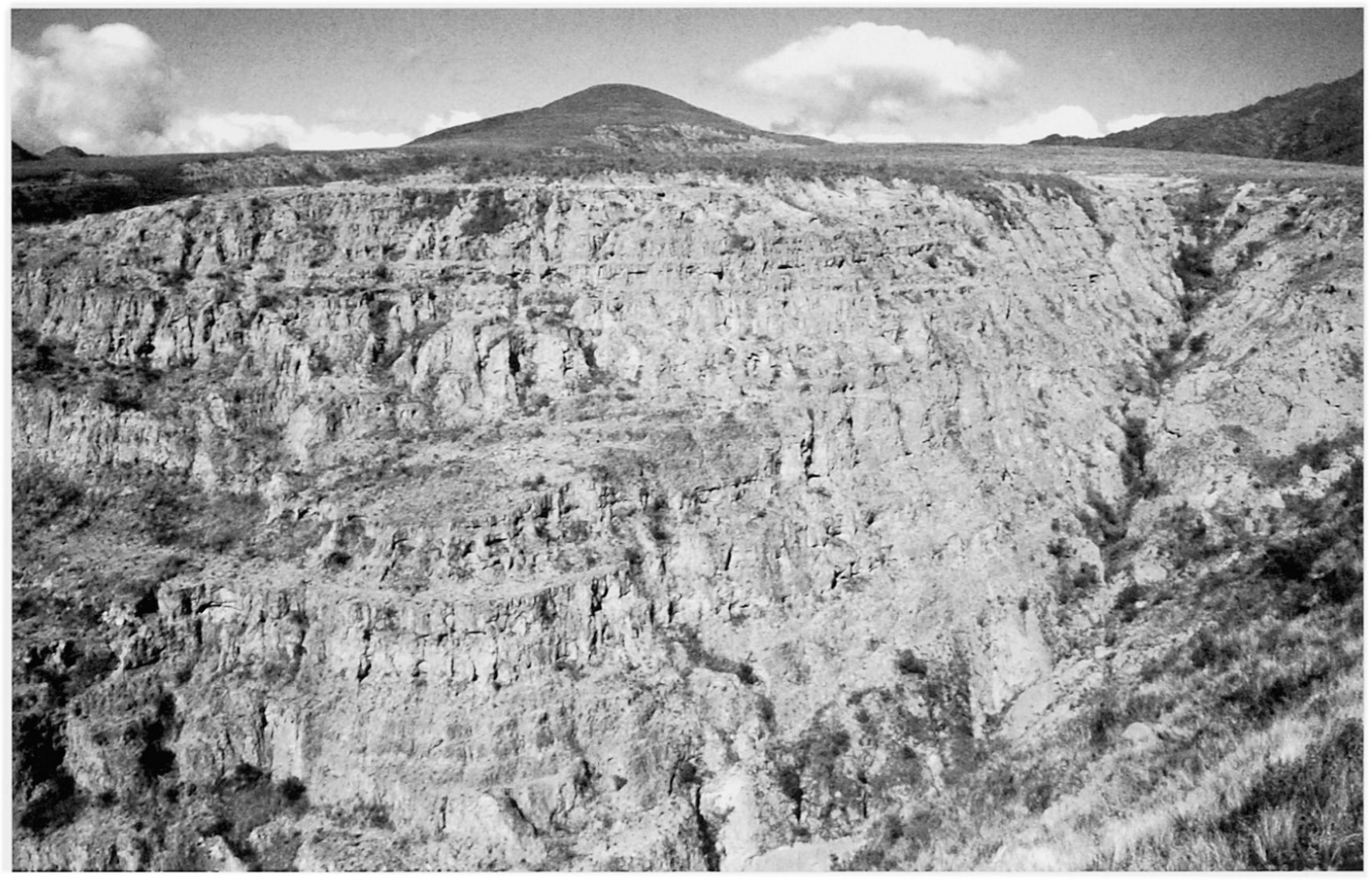

Abb. 1: Foto der oberen $40 \mathrm{~m}$ des Las Carreras-Profils in Valle de Tafí

Photograph of the upper $40 \mathrm{~m}$ of Las Carreras sequence in Valle de Tafi

Photographie de la partie sommitale $(40 \mathrm{~m})$ de la série de Las Carreras dans la Valle de Tafí

Foto: A. SCHELLENBERGER

sind in SCHELlenberger (2004) ausführlich beschrieben. Die geochronologische Einordnung der Sedimente stützt sich auf paläomagnetische Messungen (SCHELLENBERGER et al. 2003), AMS- und konventionelle ${ }^{14} \mathrm{C}$-Alter sowie auf Lumineszenz-Datierungen polymineralischer Feinkornproben $(4-10 \mu \mathrm{m})$ mit der multiple-Aliquot-Regenerationsmethode (SCHELLENBERGER 2004).

\section{Das Becken von Tafi: eine Sedimentfalle in der Ostabdachung der Tucumaner Anden}

Der Landschaftsraum Nordwestargentiniens wird geprägt durch den starken Gegensatz zwischen der weitläufigen Tiefebene des Gran Chaco und dem Gebirgskomplex der Zentralen Anden (Abb. 2). In der Provinz Tucumán, wo die ansonsten vorgeschalteten subandinen Sierren nach Süden hin aussetzen, steigen die Gebirgszüge der Präpuna unvermittelt aus dem Tiefland empor. Innerhalb kurzer Entfernung wird ein Reliefunterschied von bis zu 5000 Metern erreicht.
Sowohl das Klima als auch die Vegetation zeichnen in einem W-E-Transekt die topographischen Verhältnisse nach (Abb. 3). Die über Nordargentinien ausgeprägte zonale Abnahme der Jahresniederschläge von Ost nach West wird etwa $60 \mathrm{~km}$ östlich des Gebirgsrandes (Station Las Cejas) durch eine Niederschlagszunahme unterbrochen. Diese hygrische Anomalie kulminiert an der Ostabdachung der Anden, wo die Niederschläge zwischen 1000 und $2500 \mathrm{~mm}$ erreichen, und endet mit einer scharfen klimatischen Grenze auf einer Höhe zwischen 1500 und 2000 m ü.M. (vgl. Station Valle de Tafí). Die Spannbreite der potentiellen natürlichen Vegetation reicht von den xerophilen bis mesophilen Chaco-Wäldern über die immergrünen Wälder des Tucumaner Bergfeuchtwaldes (Yungas im engeren Sinne: Laurel- und Myrtaceen-Wald) bis zu den laubabwerfenden Wäldern der höheren Berglagen (Queñoa-, Aliso- und Nogal-Pino-Wald). Grassteppen, wie sie im semiariden Becken von Tafí die Vegetationsstruktur dominieren, leiten zu den hochandinen Vegetationseinheiten über. Im Talraum um Santa María herrscht halbwüstenartige Vegetation 


\begin{tabular}{|c|c|c|c|c|}
\hline $\begin{array}{l}\text { Profil (Anzahl Paläo- } \\
\text { böden / Mächtigkeit) }\end{array}$ & Situation & $\begin{array}{l}\text { GPS- } \\
\text { Koordinaten }\end{array}$ & $\begin{array}{c}\text { Höhe }^{(a)} \\
\text { Exposition }\end{array}$ & Topoklima $^{(\mathrm{b})}$ \\
\hline $\begin{array}{l}\text { Las Carreras } \\
(32 / 50 \mathrm{~m})\end{array}$ & $\begin{array}{l}\text { Gully; } \\
\text { Löss-Plateau }\end{array}$ & $\begin{array}{l}26^{\circ} 56^{\prime} 38.5^{\prime \prime} \mathrm{S} \\
65^{\circ} 45^{\prime} 57.6^{\prime \prime} \mathrm{W}\end{array}$ & $\begin{array}{l}2290 \\
\mathrm{~N}-\mathrm{NE}\end{array}$ & $\begin{array}{l}\text { fresco- } \\
\text { húmedo }\end{array}$ \\
\hline $\begin{array}{l}\text { La Angostura } \\
(7 / 14.6 \mathrm{~m})\end{array}$ & $\begin{array}{l}\text { arenaartige Grube; } \\
\text { neotektonisch gehobener } \\
\text { Hangfuss }\end{array}$ & $\begin{array}{l}26^{\circ} 56^{\prime} 39.2^{\prime \prime} \mathrm{S} \\
65^{\circ} 40^{\prime} 04.1^{\prime \prime} \mathrm{W}\end{array}$ & $\begin{array}{l}1895 \\
\text { NNE / W }\end{array}$ & $\begin{array}{l}\text { fresco- } \\
\text { húmedo }\end{array}$ \\
\hline $\begin{array}{l}\text { El Rincón } \\
(12 / 35.8 \mathrm{~m})\end{array}$ & $\begin{array}{l}\text { Strassenanschnitt; } \\
\text { Ostabdachung der } \\
\text { Sierra de Aconquija }\end{array}$ & $\begin{array}{l}26^{\circ} 57^{\prime} 36.2^{\prime \prime} \mathrm{S} \\
65^{\circ} 46^{\prime} 29.3^{\prime \prime} \mathrm{W}\end{array}$ & $\begin{array}{l}2315 \\
\text { E-SSE }\end{array}$ & $\begin{array}{l}\text { frío- } \\
\text { húmedo }\end{array}$ \\
\hline $\begin{array}{l}\text { El Dique } \\
(13 / 21.8 \mathrm{~m})\end{array}$ & $\begin{array}{l}\text { linearer Anschnitt; } \\
\text { quartäre Beckenfüllung über } \\
\text { muldenförmigem Basement }\end{array}$ & $\begin{array}{l}26^{\circ} 55^{\prime} 56.0^{\prime \prime} \mathrm{S} \\
65^{\circ} 41^{\prime} 17.1^{\prime \prime} \mathrm{W}\end{array}$ & $\begin{array}{l}1925 \\
\text { N-NNE }\end{array}$ & $\begin{array}{l}\text { templado- } \\
\text { seco }\end{array}$ \\
\hline
\end{tabular}

(a) Profil-Top [m ü. M.]; ${ }^{\text {(b) }}$ nach Barbieri de Santamarina \& Rohmeder (1947): fresco = frisch, frío = kalt; templado $=$ mild, húmedo $=$ feucht, seco $=$ trocken

Tab. 1: Bearbeitete Löss-Paläoboden-Sequenzen im Valle de Tafí

Loess-paleosol sequences studied in Valle de Tafí

Séries des paléosols de loess dans la Valle de Tafi

Quelle: SCHELLENBERGER 2004

vor, worin der Regenschatteneffekt der Gebirgsketten am östlichen Andenrand eindrucksvoll zum Ausdruck kommt (Abb. 3).

Das intramontane Becken Valle de Tafí $\left(27^{\circ} \mathrm{S}, 66^{\circ} \mathrm{W}\right.$; 1850 bis $2500 \mathrm{~m}$ ü.M.) befindet sich $50 \mathrm{~km}$ westlich der Provinzhauptstadt San Miguel de Tucumán (Abb. 2). Es umfasst eine Fläche von etwa $150 \mathrm{~km}^{2}$ und liegt im Anstieg zur argentinischen Vorpuna, eingebettet zwischen der Sierra de Aconquija im Süden, den Cumbres Calchaquíes im Norden sowie den Cumbres Mala Mala im Osten. Der 3042 m ü.M. gelegene Abra de Infiernillo bildet im Nordwesten den Übergang zum Becken von Santa María, das mit den nordwärts anschliessenden Valles Calchaquíes ein mehrere Hundert Kilometer langes, markantes Längstal bildet (Abb. 2). Im Süden begrenzt das isolierte Massiv des Ñuñorco Grande (3321 m ü.M.) das Valle de Tafí. Eine mächtige Bruchscholle, der $2680 \mathrm{~m}$ ü.M. erreichende Cerro Pelao, teilt das Becken in zwei ungleich grosse Bereiche. Die Entwässerung des gesamten Einzugsgebietes erfolgt durch den Río Tafí östlich des Ñunorco Grande bei La Angostura. Dort endet der flache Talboden und die steilen, tief zertalten Hänge der östlichen Andenabdachung setzen ein. Eine zweite Öffnung hin zum präandinen Vorland stellt der Passübergang in die Quebrada del Portugués dar, welcher sich zwischen dem Nuñorco Grande und der Sierra de Aconquija im Südwesten des Talraums befindet (Abb. 2).
Aufgrund der topographischen Verhältnisse fungiert das Becken von Tafí als Sedimentfalle. Quartäre Sedimente, darunter zu einem wesentlichen Teil primäre Lösse und Lössderivate, überlagern in wechselnder Mächtigkeit von bis zu über 50 Metern (vgl. Abb. 1) das bewegte Basement aus präkambrischen Metamorphiten und ordovizischen Granitoiden (GIANFRANCISCO et al. 1998), welches sporadisch an die Oberfläche tritt.

\section{Die klimatischen Verhältnisse an der Ostabdachung der Tucumaner Anden}

\subsection{Das südamerikanische Monsunsystem (SAMS)}

Dass über dem südamerikanischen Kontinent ein Monsunsystem existiert, ist eine vergleichsweise junge Erkenntnis. Vor ZHou \& LAU (1998) waren die grossräumigen Luftströmungen nicht in dieser Weise als genetisch zusammengehörendes, monsunales System verstanden worden, wie es in Abbildung 4 modellhaft dargestellt ist: Im Südsommer treibt ein Hitzetief über dem Gran Chaco, verstärkt durch ein kleineres thermisch-orographisches Tiefdrucksystem über Nordwestargentinien (Seluchi et al. 2003), die monsunale Zirkulation an. Entlang der Andenostabdachung transportiert ein low level jet tropische Luftmassen nach Süden. Die darin gespeicherte Feuchtigkeit gelangte ursprünglich mit dem Nordost-Passat aus dem tropischen Atlantik nach Amazonien, wo sie im 


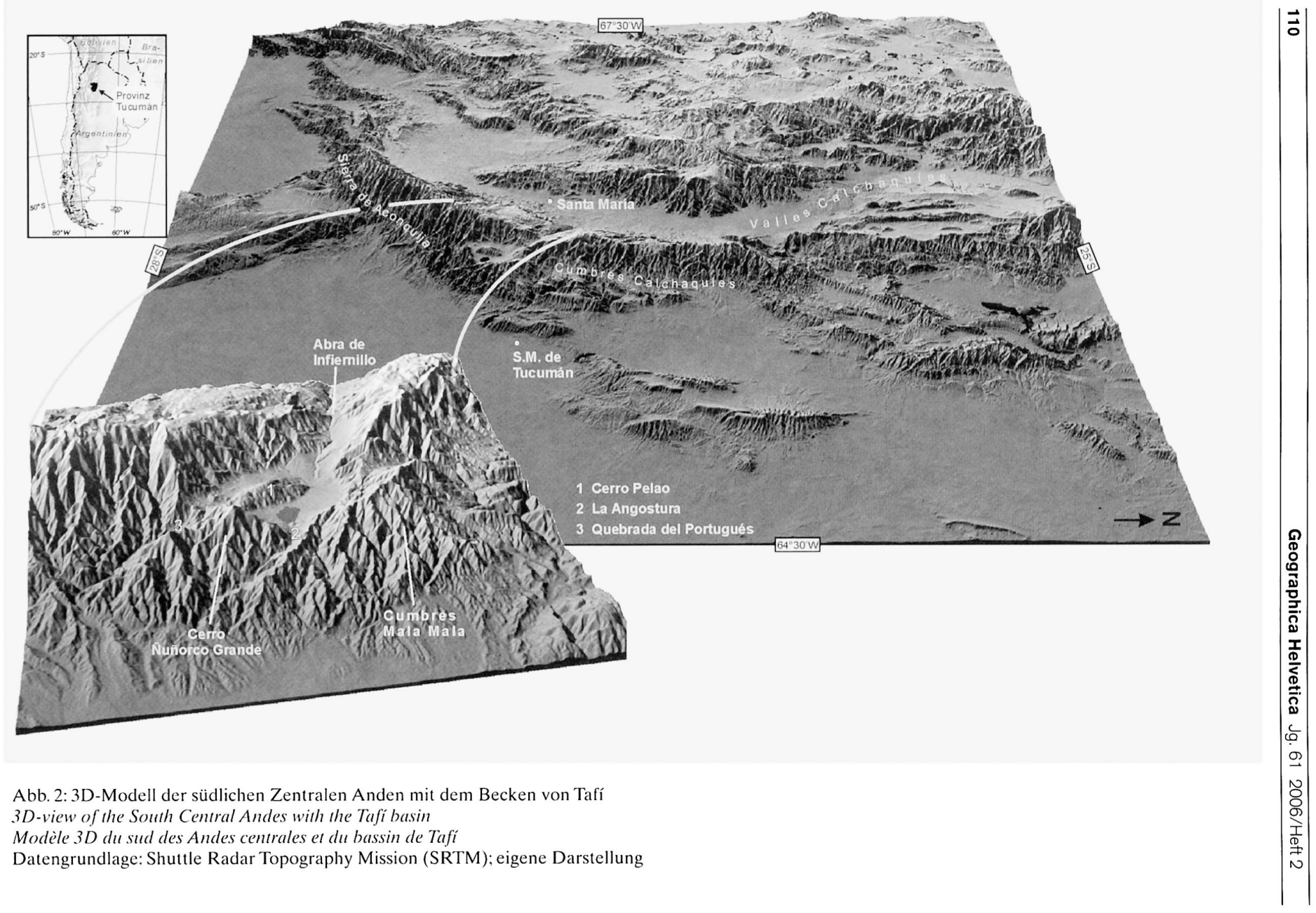



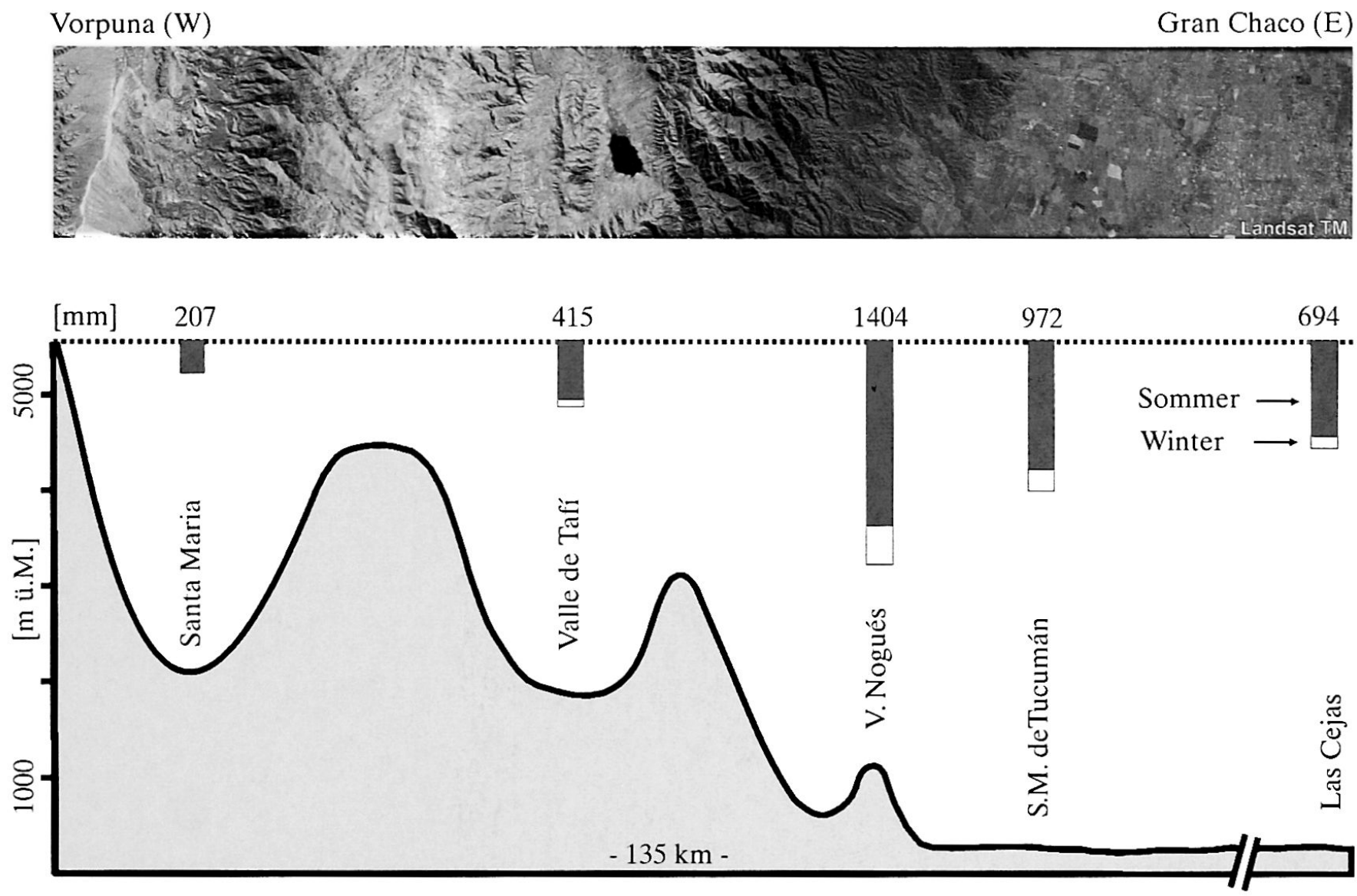

Abb. 3: W-E-Transekt der Jahresniederschläge und der Vegetationsbedeckung in Abhängigkeit vom Relief auf der geographischen Breite des Beckens von Tafí $\left(27^{\circ} \mathrm{S}\right)$

Cross section of the annual precipitation and of the vegetation cover as a function of topography at $27^{\circ} S$

Transect ouest-est des précipitations annuelles et de la couverture végétale en fonction du relief du bassin de Tafí $\left(27^{\circ} \mathrm{S}\right)$

Quellen: Bianchi \& Yañez 1992; Endlicher 1995; Minetri 1999; Landsat TM, 8/1986

Laufe des Weitertransports mehrfach rezykliert wurde (Nogues-Paegle et al. 2002). In der Provinz Tucumán entlädt sich die feuchtwarme Luft mehrheitlich in heftigen konvektiven Niederschlägen (Gewittern), wobei der durch das abrupte Ansteigen der Vorpuna bedingte orographische Effekt niederschlagsverstärkend wirkt (vgl. Abb. 2 und 3; Endlicher 1995). Bisweilen werden diese konvektiven Sommerregen von aus Patagonien stammenden Kaltfronten ausgelöst und/oder verstärkt (GarReaud 2000).

Etwa $90 \%$ des Jahresniederschlags im Vorland der Tucumaner Anden gehen auf den monsunalen Sommerniederschlag zurück. Der restliche Feuchteeintrag entstammt dem subtropischen Atlantik (MARENGO et al. 2004). Dessen Bedeutung wird durch den Dipolmodus zwischen dem low level jet und der Südatlantischen Konvergenzzone gesteuert (Barros et al. 2002). Ein Teil des geringen Winterniederschlags hat schlies- slich seinen Ursprung im Pazifik (Garreaud 2000; Vuille \& Ammann 1997).

\subsection{Topoklimate im Becken von Tafí}

Im Valle de Tafí gibt es erhebliche standortkundliche Unterschiede, die primär in einer hohen topoklimatischen Variabilität begründet liegen (BARBIERI dE Santamarina \& Rohmeder 1947). Der in der wissenschaftlichen Literatur etwas in Vergessenheit geratene Begriff «Topoklima» drückt prägnant die Abhängigkeit des Mesoklimas von den Reliefverhältnissen aus und wird aus diesem Grunde hier aufgegriffen. Klimatope (die räumliche Ausprägung der verschiedenen Topoklimate) im Becken von Tafí können kleinräumig begrenzt sein und abrupt innerhalb von wenigen Metern einsetzen (z.B. in engen Tälern, Gullies) oder Dimensionen von mehreren Quadratkilometern einnehmen und kontinuierlich ineinander übergehen (z.B. auslaufender Regenschat- 


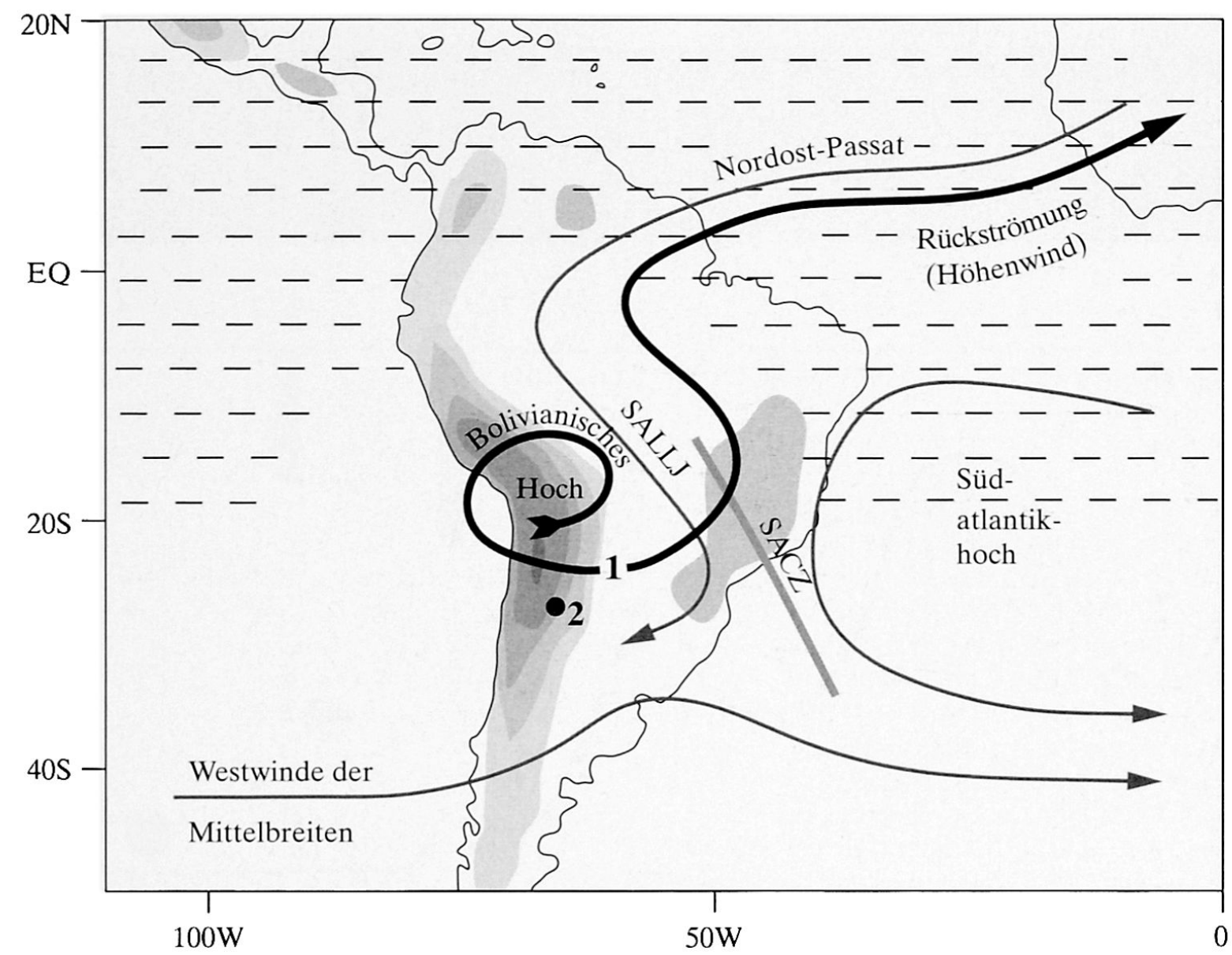

Abb. 4: Konzeptionelles Modell des südamerikanischen Monsunsystems. Gegenden mit vorherrschenden Ostwinden in der unteren Troposphäre sind gestrichelt. (1) Gran Chaco Tief; (2) nordwestargentinisches Tief; $(\bullet)$ Valle de Tafí $\left(27^{\circ} \mathrm{S}, 66^{\circ} \mathrm{W}\right)$; SALLJ = südamerikanischer low level jet (bodennaher NNW-Wind entlang der Ostabdachung der Anden); SACZ = Südatlantische Konvergenzzone

Conceptual model of the South American monsoon system. The areas where low level easterlies prevail are indicated by dashed lines. (1) Gran Chaco low; (2) NW-Argentinian low; (•) Valle de Tafi $\left(27^{\circ} S, 66^{\circ} \mathrm{W}\right) ; S A L L J=$ South American low level jet (north-northwesterlies along the eastern side of the Andes); SACZ = South Atlantic convergence zone

Modèle conceptuel du système de mousson sud-américain. Les zones où les vents d'est de basse altitude dominent sont indiquées par des lignes discontinues. (1) Dépression du Gran Chaco; (2) Dépression du nord-ouest argentin; (•) Valle de Tafí $\left(27^{\circ} S, 66^{\circ} \mathrm{W}\right) ; S A L L J=J e t$ de basse altitude sud-américain (vent NNW qui souffle à l'est des Andes); $S A C Z=$ Zone de convergence de l'Atlantique Sud

Quellen: ZHOU \& LAU 1998, leicht verändert; Graphik: L. BAUMANN

teneffekt eines Berges). Sie spiegeln sich in der Vegetation (Artzusammensetzung, Deckungsgrad), der Landnutzung und sogar der Besiedlungsgeschichte wider (Rohmeder 1955). Auch in den Oberflächenböden variiert, oft innerhalb geringer Entfernung, eine Vielzahl pedologischer Merkmale (Schellenberger 2004): z.B. die Mächtigkeit der Horizonte, der Grad der Tonanreicherung, die Stärke und Art der Aggregierung, die Existenz von Ton-Humus-Überzügen auf den Aggregaten und das Ausmass der sekundären Kalkanreicherung. Entlang eines Gradienten der
Netto-Bodenfeuchte sind auf Löss alle Übergänge vom Albic Luvisol (A-E-Bt) bis zum Calcic Kastanozem $(\mathrm{Ak}-\mathrm{Ck})$ vertreten.

Die Niederschlagsereignisse im Valle de Tafí stehen im genetischen Zusammenhang mit den meteorologischen Prozessen im Andenvorland, sie sind jedoch in ihrer Intensität erheblich abgeschwächt (vgl. Abb. 3; Hunzinger 1995; Maldonado 1995). Deutlich über 90\% des Jahresniederschlags fallen hier im Südsommer, meist nachmittags und nachts, in Form von lang- 
anhaltenden und flächendeckenden (Niesel-)Regen. Starkregen sind die Ausnahme, und lediglich Gewitter führen gelegentlich zu örtlich begrenzten Schauern. Die Ursache für die Herausbildung der verschiedenen Klimatope im Talraum von Tafí muss demnach primär im Niederschlagsmuster während des trockenen Winterhalbjahrs zu suchen sein.

Im Winterhalbjahr (April bis September) ist die potentielle Evapotranspiration aufgrund der intensiven Sonneneinstrahlung deutlich höher als die verfügbare Niederschlagsmenge aus den zumeist feinen Nieselregen. Daraus resultiert ein erhebliches Bodenfeuchtedefizit. Der wichtigste Feuchteeintrag erfolgt unter diesen Rahmenbedingungen durch Wolkennebel, die aperiodisch im Abstand weniger Tage aus dem tiefer gelegenen Bergregenwald auf topographisch vorgegebenen Pfaden in das Becken einströmen (Fotos typischer Konstellationen bei La Angostura und oberhalb der Quebrada del Portugués (vgl. Abb. 2) finden sich in SCHELlenderger 2004: 106-107). Die tageszeitliche Dynamik der Wolken und Nebel sowie ihre Zugbahnen sind derart charakteristisch, dass sie bereits früh beschrieben wurden (RAQUela GIMÉNEZ 1950; SAJAREVICH 1950). Abgesetzte, vor allem aber durch die Vegetation abgefangene Nebelniederschläge lassen auf diese Weise ein Mosaik aus Klimatopen mit unterschiedlichem (Boden-)Wasserregime entstehen. Die topographische Lage (Exposition, Höhe, Hangneigung) bestimmt die Empfänglichkeit für Nebelniederschlag und moduliert ihrerseits zusammen mit anderen Faktoren, wie den Substrateigenschaften und der Vegetation, den thermischen und hygrischen Haushalt eines Standorts. Je grösser die Oberfläche der Pflanzendecke, welche sie dem Nebelfluss entgegenrichtet, desto grösser ist der Interzeptionsgewinn. Diese positive Rückkoppelung wird durch das Vorkommen von Bartflechten auf manchen Bäumen eindrucksvoll illustriert.

Die Nebelniederschläge werden bei den Standardmessungen der fest installierten Klimastationen nicht systematisch erfasst. Eine einjährige Messperiode in den Tucumaner Yungas in $1350 \mathrm{~m}$ ü.M. hat ergeben, dass ihr Anteil am Feuchteeintrag des Gesamtjahres bei $28 \%$ liegt ( $470 \mathrm{~mm}$ von $1696 \mathrm{~mm})$. Im Winterhalbjahr werden sogar $59 \%$ erreicht ( $308 \mathrm{~mm}$ von $522 \mathrm{~mm}$; HUNZINGER 1997). Es ist anzunehmen, dass der relative Anteil der Nebelniederschläge im Winter mit der Höhe (und damit stark abnehmendem Regenniederschlag, vgl. Abb. 3) weiter steigt und - geeignete Vegetation zum Auskämmen der feinen Nebeltröpfchen vorausgesetzt - sukzessive zum dominierenden Faktor wird. Der Betrag der effektiven Winterniederschläge auf den diesbezüglich begünstigten Flächen im Valle de Tafí dürfte folglich klar höher sein als in Abbildung 3 angegeben.

\section{Indizien für einen Paläomonsun}

In der spärlichen Literatur zur Entwicklung der grossräumigen atmosphärischen Zirkulation in Südamerika gibt es klare Hinweise darauf, dass das gegenwärtig über Nordwestargentinien beobachtete Muster mit einer aus dem Atlantik stammenden Feuchte über die letzten Millionen Jahre hinweg im Wesentlichen Bestand hatte. Ohne weitere Details zu nennen, gibt IRIONDO (1993) ein miozänes Alter an. Auf der Grundlage von Fazies-Analysen an miozänen Sedimenten in den südlichen Zentralen Anden postulieren STARCK \& Anzótegui (2001) die Existenz feuchter Ostwinde seit ca. 10-9 Mio. Jahren. Die sukzessive Verlagerung der orogenetischen Prozesse in Richtung Osten (Heraushebung der Zentralen Anden) zog eine gleichgerichtete Verschiebung der Bereiche mit orographischen Niederschlägen nach sich. Der einsetzende Regenschatten-Effekt im Becken von Santa María (Abb. 2) ist anhand von isotopengeochemischen Untersuchungen zwischen 3 und 2.5 Ma belegt (KLEINERT \& STRECKer 2001). Dies fällt mit dem letzten und entscheidenden Hebungsimpuls der Sierra de Aconquija und der Cumbres Calchaquíes während der spätpliozänen-frühpleistozänen Diaguita-Phase zusammen. Damit wurden die feuchten Ostwinde von wirksamen Barrieren geblockt und zugleich das rezente Niederschlagsregime der Vorpuna in seinen Grundzügen etabliert. Bei ihren Untersuchungen der pleistozänen Schneegrenzen in den Zentralen Anden kommen Fox \& Strecker (1991) und Haselton et al. (2002) übereinstimmend zu der Ansicht, dass der wiederholte Eisaufbau trotz dieser orographischen Barrieren durch einen erhöhten atlantischen Feuchteeintrag ermöglicht wurde. Die persistente Luv- und Leelage hat zudem im Relief der Andenostabdachung weitere Spuren hinterlassen: Steilen, tief zertalten Ostflanken stehen auf der Westseite flacher geneigte, kaum eingeschnittene Hänge mit deutlich geringerer Reliefenergie gegenüber (Abb. 2).

Der entscheidende Hinweis, dass dieser atlantische Feuchteeintrag in Nordwestargentinien auch in früheren Zeiten eine monsunale Dynamik mit Sommerregen und Wintertrockenheit aufwies, stammt aus dem Becken von Tafí. Zusätzlich zum Las Carreras-Profil wurden dort drei weitere Löss-Paläoboden-Sequenzen aus unterschiedlichen Klimatopen bearbeitet: die Profile La Angostura, El Dique und El Rincón (Tab. 1; Abb. 5; SCHELlenderger 2004). Die topoklimatische Untergliederung des Beckens von Tafí nach BarbierI De Santamarina \& Rohmeder (1947; Tab. 1) hat sich im Laufe der Geländearbeiten als eine gute Charakterisierung der Profilstandorte erwiesen. Obwohl beispielsweise die Distanz zwischen La Angostura («fresco-húmedo») und El Dique («templado-seco») weniger als drei Kilometer beträgt, sind dort im glei- 


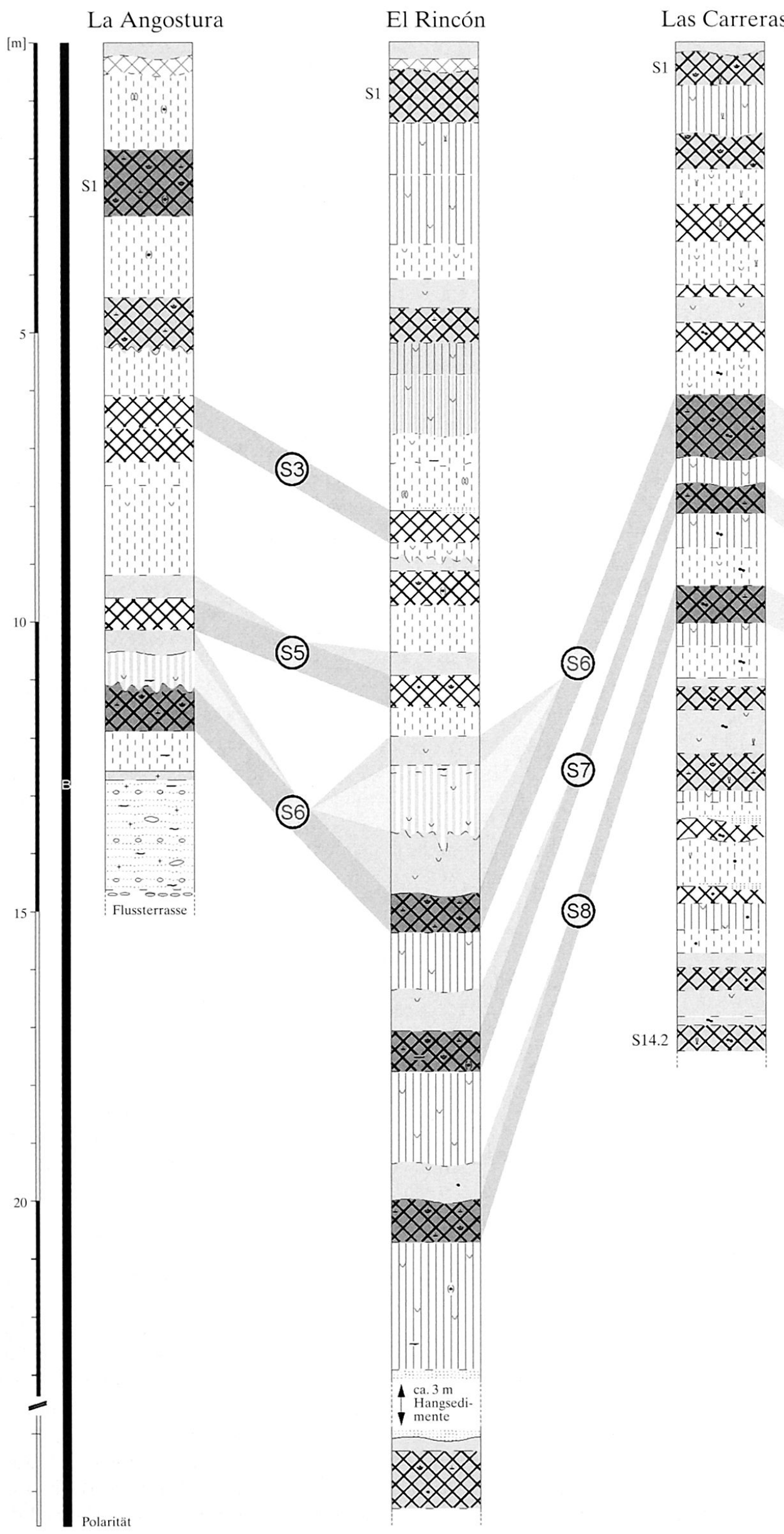

El Dique

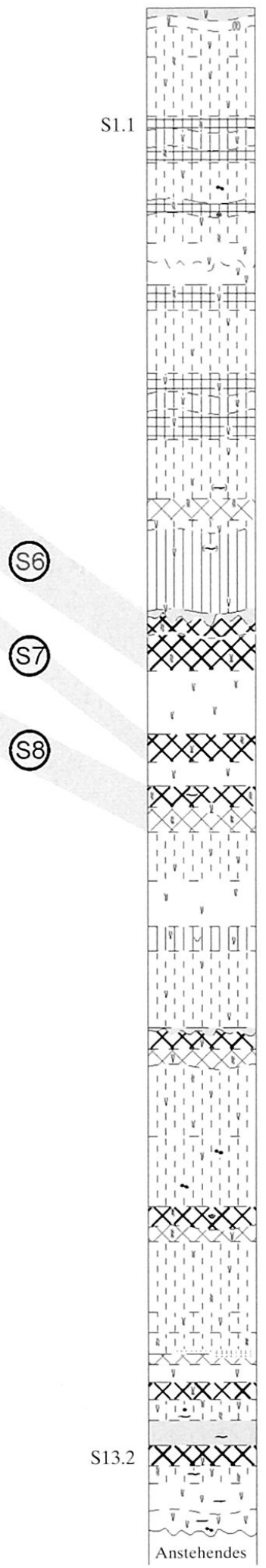


Horizonte

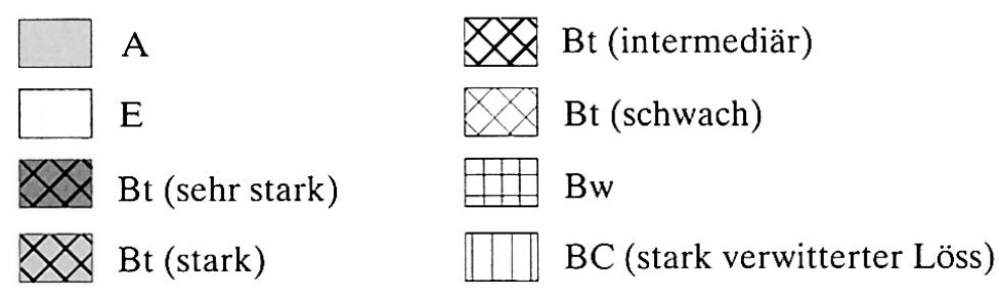

CB (schwach verwitterter Löss)

C (Löss)

Gekappter Bt-Horizont

Ausgangsmaterial verspült

\section{Pedofeatures}

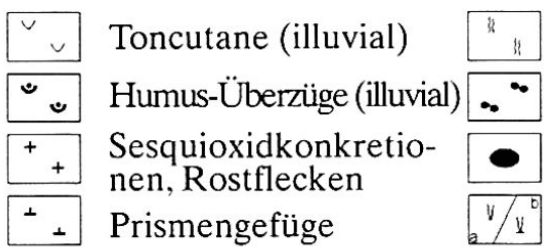

Pseudomycelien

Carbonatkonkretionen

Lösskindl

Sekundärcarbonat

a) in der Bodenmatrix

b) auf Aggregat-/Rissoberflächen
Horizontgrenzen

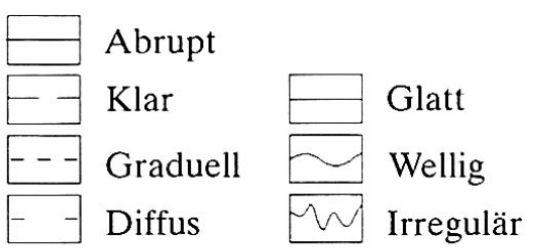

\section{Lithologie}

In Feinsediment eingelagerte Kiese
Terrassenschotter

Abb. 5: Pedostratigraphische Korrelation der Löss-Paläoboden-Sequenzen La Angostura, El Rincón, Las Carreras und El Dique. Die relative Stärke der Bodenbildung in den B-Horizonten wird durch unterschiedliche Signaturen veranschaulicht. S = Paläoboden; B = Brunhes-Epoche. Für Details vgl. Schellenberger (2004).

Pedostratigraphic correlation of the loess-paleosol sequences La Angostura, El Rincón, Las Carreras and El Dique. The relative degree of pedogenesis in the B horizons is indicated by different patterns. $S=$ paleosol, $B=$ Brunhes chron. For details see SCHELlENBERGER (2004).

Corrélation pédostratigraphique des séries de paléosols de loess de La Angostura, El Rincón, Las Carreras et El Dique. Le degré relatif de pédogenèse dans les horizons $B$ est indiqué par différents figurés. $S=$ paléosol, $B=$ chron de Brunhes. Voir SchellenBerger (2004) pour détails.

chen Ausgangsmaterial vollkommen unterschiedliche Oberflächenböden ausgebildet: ein mässig entwickelter Haplic Luvisol (A-Bt) am ersten bzw. ein Calcic Kastanozem (Ak-Ck) am zweitgenannten Standort. In den Sedimentkörpern, die magnetostratigraphisch der Brunhes-Epoche zugerechnet werden können, sind in La Angostura 7, in El Rincón 12 und in El Dique 13 Paläoböden eingeschaltet. Eine ausführliche Beschreibung der stratigraphischen Verhältnisse und der paläopedologischen Eigenschaften ist in SCHEllenberger (2004) enthalten. Dort wird auch im Detail erläutert, wie die Profil-Abfolgen trotz ihrer zum Teil sehr unterschiedlichen visuellen Ausprägung anhand von Leithorizonten miteinander korreliert werden können (Abb. 5: vgl. den Bt-Horizont des am stärksten entwickelten Paläobodens S6 bzw. die markante Dreierfolge S6-S7-S8.). Eine Synthese der vier
Pedostratigraphien, die jeweils überregionale Klimaveränderungen als auch standortspezifische Einflüsse (Topoklimate) widerspiegeln, erlaubt eine präzisere paläoklimatische Interpretationen für das Becken von Tafí, als dies auf der Grundlage eines Einzelprofils möglich wäre.

Für die in diesem Artikel verfolgte Fragestellung, ob das Profil Las Carreras ein Langzeitklimaarchiv für den Paläomonsun darstellt, ist die Beobachtung massgeblich, dass alle vier Löss-Sequenzen im Tiefenprofil die dem jeweiligen Klimatop entsprechende (pedo)fazielle Ausprägung bewahren. Als besonders anschauliche Belege hierfür seien die Mächtigkeit der Bt-Horizonte in La Angostura und im Vergleich dazu in El Dique, die durchgehende sekundäre Kalkanreicherung in El Dique und der gute Erhaltungsgrad der 
humosen A-Horizonte sowie der hohe Verwitterungsgrad der mächtigen Löss-Pakete in El Rincón genannt (vgl. Abb. 5). Nicht nur die Oberflächenböden, sondern auch die fossilen Böden und zwischenlagernden Lösse zeigen folglich über alle Intensitätsschwankungen vergangener Feucht- und Trockenphasen hinweg einen Bezug zum jeweiligen Standort, der eine Abgrenzung der vier Profile untereinander ermöglicht. Dies kann nur in dem Sinne interpretiert werden, dass im betreffenden Zeitraum grundsätzlich ähnliche Standortunterschiede (Paläoklimatope) wie rezent bestanden haben müssen. Dies wiederum bedingt - gemäss den in Kapitel 4.2 angestellten Überlegungen - die Existenz von topographisch gesteuerten und deswegen kleinräumig diversifizierenden Nebelniederschlägen während des trockenen Winterhalbjahrs. Letztere treten im Becken von Tafí nur bei einem monsunal geprägten Sommerregenregime konsistent auf. Ginge beispielsweise die Bodenbildung S1 auf einen verstärkten Feuchteeintrag aus der Westwindzone zurück, wäre es aufgrund des dann flächendeckenden winterlichen Regenniederschlags nicht zur Ausbildung der markanten pedologischen Unterschiede dieses Paläobodens zwischen den einzelnen Profilen gekommen (vgl. Abb. 5). Aus dem Las Carreras-Profil, für welches ein «getuntes» Altersmodell vorliegt (SCHELlenberger et al.), lässt sich damit die Existenz des südamerikanischen Monsunsystems für mindestens die letzten $600 \mathrm{ka}$ ableiten.

In Las Carreras («fresco-húmedo») zeichnet sich mit Unit II (S18 bis S28) zwischen ca. 0.72 Ma und 1.01 Ma eine deutliche Änderung der (pedo)faziellen Verhältnisse ab (Schellenberger \& VeIT 2006; dieser Profilteil ist in Abb. 5 nicht dargestellt). Sowohl die Paläoböden als auch die Löss-Lagen gleichen sich der in El Dique ausgeprägten «templado-seco»-(Pedo)Fazies an. Unit III (S29-S32) im Liegenden entspricht wieder weitgehend der insgesamt klar feuchteren Unit I (S1-S17). $\mathrm{Da}$ in keinem der drei anderen Profile die stratigraphische Position S18 erreicht wird, entfällt die direkte Vergleichbarkeit eines konsistenten Verhaltens der Paläoklimatope untereinander nach obigem Muster. Auffällig ist die zeitliche Übereinstimmung von Unit II mit der MPT. Ein Vergleich mit den Feuchteverhältnissen in Amazonien deutet darauf hin, dass das Monsunsystem während dieser Zeit des globalen Klimasystemwandels Bestand hatte und die Umweltveränderungen am Profilstandort Las Carreras auf einen signifikant reduzierten Feuchtetransport des low level jet zurückgehen (SCHELLENBERGER et al.).

\section{Fazit}

Geologische und geomorphologische Untersuchungen deuten einstimmig darauf hin, dass in Nordwestargentinien die grossräumige atmosphärische Zirkulation das gesamte Quartär über durch atlantische Luftmassen geprägt war. Dieser Literaturbefund wird im Becken von Tafí durch den Vergleich der Pedostratigraphien von vier bearbeiteten Löss-Profilen gestützt. Der Nachweis, dass nicht nur die Oberflächenböden, sondern auch Paläoböden und Lösse die für das jeweilige Klimatop charakteristische pedo(fazielle) Ausprägung aufweisen, belegt zudem, dass eine mit der heutigen Situation vergleichbare monsunale Dynamik vorherrschte. Sommerregen lieferten das Gros des Jahresniederschlags. Ihr Gesamtbetrag bestimmte langfristig das Auftreten von Feucht- oder Trockenphasen und damit entweder die Intensität von Bodenbildungen oder die Mächtigkeit von Löss-Ablagerungen. Im trockenen Winterhalbjahr sorgten Nebelniederschläge relativ unabhängig vom Umfang des sommerlichen Feuchtetransports für einen kleinräumig diversifizierten Feuchteeintrag und prägten auf diese Weise gleichermassen das standortspezifische Erscheinungsbild von Paläoböden wie Lössen (Paläoklimatope).

Auf dieser Datengrundlage muss offenbar nicht einmal Ockham's Razor bemüht werden, um zu dem Schluss zu gelangen, dass die Löss-Paläoboden-Sequenz Las Carreras das erste bekannte Langzeitklimaarchiv für das südamerikanische Monsunsystem darstellt. Begünstigt durch die klimasensitive Lage des Beckens von Tafí ist in den Lössen die Dynamik des SAMS während der letzten 1.2 Ma abgebildet. Erstmals sind im südhemisphärischen Teil des Subkontinents Rückschlüsse auf langfristige Steuerungsmechanismen während des Quartärs und auf die Umweltveränderungen im Zuge der MPT möglich. Da trotz einer Vielzahl neuer Erkenntnisse (z.B. CooK \& Vizy 2006; MARENGO et al. 2004) ein schlüssiges Paläomonsun-Modell, wie es beispielsweise für das chinesische Löss-Plateau existiert (Liu \& Ding 1998), nicht in Sicht ist, unterliegen paläoklimatische Rekonstruktionen auf der orbitalen Zeitskala allerdings bis auf weiteres erheblichen Schwierigkeiten. Unabhängig davon dürfte sich - spätestens mit der Validierung adäquater Klimaproxies, welche derzeit noch nicht zur Verfügung stehen (SCHELLENBERGER \& Verr 2006) - die Las Carreras-Sequenz zukünftig als Standardprofil in Südamerika etablieren.

\section{Literatur}

Barbieri de Santamarina, E. \& G. Rohmeder (1947): Deducción de topoclimas en el Valle de Tafí por medio de la vegetación autóctona. - In: Gómez OMil, D., Barbieri de Santamarina, E. \& G. Rohmeder (Hrsg.): Tres contribuciones a la climatogeografía de Tucumán. - = Serie Monográfica 9, Universidad Nacional de Tucumán, Instituto de Estudios Geográficos: 17-27.

Barros, V., Doyle, M., González, M., Camilloni, I., Bejarán, R. \& R.M. Caffera (2002): Climate variability over subtropical South America and the South 
American monsoon: a review. - In: Meteorologica 27, 33-57.

BIANCHI, A.R. \& C.E. YAÑEZ (1992): Las precipitaciones en el Noroeste Argentino. - Salta: Instituto nacional de tecnología agropecuaria, Estación experimental agropecuaria Salta.

CoOK, K.H. \& E.K. VIZY (2006): South American climate during the Last Glacial Maximum: Delayed onset of the South American monsoon. - In: Journal of geophysical research 111: D02110.

ENDLICHER, W. (1995): Zur Witterungsklimatologie von Nordwestargentinien. - In: ENDlicher, W. \& E. Würschmidt (Hrsg.): Stadtklimatologische und lufthygienische Untersuchungen in San Miguel de Tucumán, Nordwestargentinien. - = Marburger geographische Schriften 128: 17-43.

Food and Agriculture Organization of the United Nations (FAO), International Soll Reference AND INFORMATION CENTRE (ISRIC) \& INTERNATIONAL SOCIETY OF SoIl SCIENCE (ISSS) (1998): World reference base for soil resources. - = World soil resources reports 84, Rome.

Fornari, M., Risacher, F. \& G. Feraud (2001): Dating of paleolakes in the central Altiplano of Bolivia. - In: Palaeogeography, palaeoclimatology, palaeoecology 172: 269-282.

Fox, A.N. \& M.R. StRecker (1991): Pleistocene and modern snowlines in the Central Andes (24-28 $\mathrm{S}$ ). - In: Bamberger geographische Schriften 11: 169-182. Fritz, S.C., BAKER, P.A., Lowenstein, T.K., Seltzer, G.O., Rigsby, C.A., Dwyer, G.S., TAPia, P.M., Arnold, K.K., Ku, T.-L. \& S. Luo (2004): Hydrologic variation during the last 170.000 years in the southern hemisphere tropics of South America. - In: Quaternary research 61: 95-104. GaRREAUD, R.D. (2000): Cold air incursions over subtropical South America: mean structure and dynamics. - In: Monthly weather review 128: 2544-2559.

Gianfrancisco, M., Puchulu, M.E., Durango de Cabrera, J. \& G.F. Aceñolaza (Hrsg.) (1998): Geología de Tucumán. $-=$ Tucumán: Colegio de graduados en ciencias geológicas de Tucumán.

Haselton, K., Hilley, G. \& M.R. Strecker (2002): Average Pleistocene climatic patterns in the southern Central Andes: controls on mountain glaciation and paleoclimate implications. - In: The journal of geology 110: 211-226.

Hastenrath, S.L. (1971): On the Pleistocene snow-line depression in the arid regions of the South American Andes. - In: Journal of glaciology 10:255-267.

Hooghiemstra, H. (1984): Vegetational and climatic history of the high plain of Bogotá, Colombia: a continuous record of the last 3.5 million years. $-=$ Dissertationes botanicae 79: 1-368.

Hooghiemstra, H. \& E.T.H. Ran (1994): Late Pliocene-Pleistocene high resolution pollen sequence of Colombia: an overview of climatic change. - In: Quaternary international 21: 63-80.
HUNZINGER, H. (1995): Struktur der Starkniederschläge zwischen Gran Chaco und Vorpuna. - In: ENDLicher, W. \& E. Würschmidt (Hrsg.): Stadtklimatologische und lufthygienische Untersuchungen in San Miguel de Tucumán, Nordwestargentinien. - = Marburger geographische Schriften 128: 60-77.

HUNZINGER, H. (1997): Hydrology of montane forests in the Sierra de San Javier, Tucumán, Argentina. - In: Mountain research and development 17: 299-308.

IRIONDO, M. (1993): Geomorphology and late Quaternary of the Chaco (South America). - In: Geomorphology 7: 289-303.

Kleinert, K. \& M.R. Strecker (2001): Climate change in response to orographic barrier uplift: Paleosol and stable isotope evidence from the late Neogene Santa Maria basin, northwestern Argentina. - In: Geological Society of America (GSA) Bulletin 113: 728-742.

Liv, T. \& Z. Ding (1998): Chinese loess and the paleomonsoon. - In: Annual review of earth and planetary sciences 26: 111-145.

Maldonado, M.E. (1995): Las precipitaciones en San Miguel de Tucumán y alrededores durante el período 1991-1993. - In: ENDLICHER, W. \& E. WürsCHMIDT (Hrsg.): Stadtklimatologische und lufthygienische Untersuchungen in San Miguel de Tucumán, Nordwestargentinien. - = Marburger geographische Schriften 128: 44-59.

Marengo, J.A., Soares, W.R., Saulo, C. \& M. Nicolini (2004): Climatology of the low-level jet east of the Andes as derived from the NCEP-NCAR reanalyses: Characteristics and temporal variability. - In: Journal of climate 17:2261-2280.

Minetri, J.L. (1999): Atlas climático del Noroeste Argentino. - San Miguel de Tucumán: Laboratorio Climatológico Sudamericano, Fundación Carl C: Zon Caldenius, Sede NOAA, Consejo Nacional de Investigaciones Cientificas y Técnicas (CONICET) y Departamento de Geografía de la Facultad de Filosofía y Letras de la Universidad Nacional de Tucumán.

Nogues-Paegle, J., Mechoso, C.R., Fu, R., Berbery, E.H., Chao, W.C., Chen, T.-C., Сook, K., Diaz, A.F., Enfield, D., Ferreira, R., Grimm, A.M., Kousky, V., Liebmann, B., Marengo, J., Mo, K., Neelin, J.D., Paegle, J., Robertson, A.W., Seth, A., Vera, C.S. \& J. Zhou (2002): Progress in Pan American CLIVAR research: Understanding the South American monsoon. - In: Meteorologica 27: 1-30.

Raquela Giménez, L. (1950): El clima de verano en el Valle de Tafí. - In: Geographia una et varia, Publicaciones Especiales 2, Instituto de Estudios Geográficos, Facultad de Filosofía y Letras, Universidad Nacional de Tucumán, San Miguel de Tucumán: 107-118.

ROHMEDER, G. (1955): Topoclimas y sus relaciones con relieve, vegetación y cultivos en el valle de Tafí (Montaña de Aconquija, Noroeste argentino). - In: Boletin de estudios geograficos II, 9, Instituto de Geografia, Universidad Nacional de Cuyo: 235-253. 
Sajarevich, E. (1950): Ensayo sobre el clima de invierno en el Valle de Tafí. - In: Geographia una et varia, Publicaciones Especiales 2, Instituto de Estudios Geográficos, Facultad de Filosofía y Letras, Universidad Nacional de Tucumán, San Miguel de Tucumán: 193-206.

SChellenberger, A. (2004): The NW-Argentinian loess record and its implications for climate history in South America over the past 1.2 Ma. - = Dissertation, Geographisches Institut, Universität Bern, http://www. stub.unibe.ch/download/eldiss/04schellenberger_a.pdf. Schellenberger, A. \& H. Veit (2006): Pedostratigraphy and pedological and geochemical characterization of the Las Carreras loess-paleosol sequence, Valle de Tafí, NW-Argentina. - In: Quaternary science reviews 25: 811-831.

Schellenberger, A., Heller, F. \& H. Veit (2003): Magnetostratigraphy and magnetic susceptibility of the Las Carreras loess-paleosol sequence in Valle de Tafí, Tucumán, NW-Argentina. - In: Quaternary international 106-107: 159-167.

Schellenberger, A., Veit, H. \& F. Heller: A 1.2 Ma loess-paleosol record of the South American Monsoon System. - In review.

Seluchi, M.E., Saulo, A.C., Nicolini, M. \& P. SatyaMURTY (2003): The northwestern Argentinean low: a study of two typical events. - In: Monthly weather review 131:2361-2378.

STARCK, D. \& L.M. Anzótegui (2001): The late Miocene climatic change - persistence of a climatic signal through the orogenic stratigraphic record in northwestern Argentina. - In: Journal of South American earth sciences 14: 763-774. Torres, V., Vandenberghe, J. \& H. Hooghiemstra (2005): An environmental reconstruction of the sediment infill of the Bogotá basin (Colombia) during the last 3 million years from abiotic and biotic proxies. - In: Palaeogeography, palaeoclimatology, palaeoecology 226: 127-148.

Vuille, M. \& C. Ammann (1997): Regional snowfall patterns in the high, arid Andes. - In: Climatic change 36: 413-423.

Zhou, J.Y. \& K.M. LaU (1998): Does a monsoon climate exist over South America? - In: Journal of climate 11: 1020-1040.

ZiNCK, J.A. \& J.M. SAYAGO (1999): Loess-paleosol sequence of La Mesada in Tucumán province, northwest Argentina. Characterization and paleoenvironmental interpretation. - In: Journal of South American earth sciences 12:293-310.

\section{Zusammenfassung: Die Lösse im Becken von Tafí (Nordwestargentinien) - ein Langzeitklimaarchiv für das südamerikanische Monsunsystem?}

Durch den Mangel an Langzeitklimaarchiven sind der Quartärforschung in Südamerika Grenzen auferlegt. Eine prominente Ausnahme stellt die $50 \mathrm{~m}$ mächtige Löss-Paläoboden-Sequenz Las Carreras im Becken von Tafí (nordwestargentinische Anden) dar, die 32 Feuchtphasen in den vergangenen $1.2 \mathrm{Ma}$ dokumentiert. Die Region liegt im Einflussgebiet des südamerikanischen Monsunsystems (SAMS) mit dominierenden Sommerregen. Im trockenen Winterhalbjahr führen selektive, den topographisch vorgegebenen Pfaden folgende Nebelniederschläge zu kleinräumigen und deutlichen Standortunterschieden. Diese topoklimatische Untergliederung des Beckens spiegelt sich auch in den Paläoböden und Lössen von insgesamt vier pedostratigraphisch miteinander korrelierten Löss-Profilen wider. Zusammen mit Literaturdaten, die einen Feuchtetransport aus dem Atlantik seit dem späten Miozän nahelegen, wird daraus auf die Persistenz des monsunalen Klimasystems während des Quartärs geschlossen. Mit dem ersten Langzeitklimaarchiv im südhemisphärischen Teil des Subkontinents wird eine Rekonstruktion der SAMS-Dynamik in Aussicht gestellt für einen Zeitraum, der in Südamerika bislang weitgehend tempus incognitum war.

Summary: Are the loess deposits in Tafi basin (northwestern Argentina) a long-term record of the South American Monsoon System?

One of the major obstacles to studying Quaternary climate evolution in South America is the paucity of long records. One prominent exception is the 50 -m-thick Las Carreras loess-paleosol sequence in Tafí basin (north-western Argentina) that reflects 32 climatic cycles over the past $1.2 \mathrm{Ma}$. Summertime precipitation is predominantly fed by the South American Monsoon System (SAMS). During the dry winter season fog interception causes significant site-specific differences in soil moisture conditions and is primarily controlled by topography. The complexity of topoclimatic regimes in Tafí valley is also reflected in the characteristics of paleosols and loess beds in four pedostratigraphically correlated loess sequences. Combination of paleopedological records and published data provides strong evidence that the large-scale atmospheric circulation patterns currently observed over north-western Argentina have persisted since the late Miocene and that a summer monsoon rainfall regime has been around for at least the last $600 \mathrm{ka}$. Thus, we interpret the Las Carreras loess-paleosol alternation to reflect climatic cycles primarily caused by SAMS variability. This first long-term record of paleomonsoonal variability will open up new and unexpected paleoclimatic resources for South American Quaternary research.

Résumé: Les dépôts de loess du bassin de Tafí au nordouest de l'Argentine gardent-ils une trace durable du système sud-américain de mousson?

L'étude des évolutions climatiques quaternaires de l'Amérique du Sud est handicapée par le manque de données enregistrées sur de longues périodes. La série des paléosols de loess du bassin de Tafí (nord-ouest 
de l'Argentine) qui présente 32 cycles climatiques sur une période de 1,2 Ma constitue de ce point de vue une notable exception. Les précipitations estivales y sont principalement fournies par le système sud-américain de mousson. Durant la saison sèche hivernale, des brouillards sont à l'origine de degrés d'humidité différents dans les sols en raison de la topographie. La complexité des régimes topoclimatiques de la Vallée de Tafi est également liée aux caractéristiques des paléosols et des lits de loess dans quatre séries pédostratigraphiques corrélées. La combinaison de données paléopédologiques et de résultats déjà publiés montre que les schémas de la circulation atmosphérique à grande échelle du nord-ouest argentin actuel sont similaires à ceux du Miocène tardif et que le régime de mousson estival est resté similaire durant ces dernières 600000 années. Par conséquent, les séries de paléosols de loess reflètent les cycles climatiques principalement en raison de la variabilité du système sud-américain de mousson. Les données à long terme de la variabilité des paléomoussons ouvrent de nouvelles perspectives pour la recherche quaternaire en Amérique du Sud.

Dr. Andreas Schellenberger, Geographisches Institut der Universität Bern, Hallerstrasse 12, CH-3012 Bern, Schweiz.

e-mail: schello@giub.unibe.ch

Manuskripteingang/received/manuscrit entré le 15.2.2006

Annahme zum Druck/accepted for publication/accepté pour l'impression: 14.6.2006 